\title{
Development and Validation of a Model for Predicting the Risk of Death in Patients with Acinetobacter baumannii Infection: A Retrospective Study
}

This article was published in the following Dove Press journal: Infection and Drug Resistance

Hui Zhang,' Yayun Zhao,' Yahong Zheng,' Qinxiang Kong, ,2 Na Lv, ' Yanyan Liu, ',3,4 Dongmei Zhao, ${ }^{5}$ Jiabin $\mathrm{Li}^{,}{ }^{1-4}$ Ying $\mathrm{Ye}^{\mathrm{I}}$

'Department of Infectious Disease, The First Affiliated Hospital of Anhui Medical University, Hefei, Anhui, People's Republic of China; ${ }^{2}$ Department of Infectious Diseases, The Chaohu Affiliated Hospital of Anhui Medical University, Hefei, Anhui, People's Republic of China; ${ }^{3}$ Anhui Center for Surveillance of Bacterial Resistance, Hefei, Anhui, People's Republic of China; ${ }^{4}$ Institute of Bacterial Resistance, Anhui Medical University, Hefei, Anhui, People's Republic of China;

${ }^{5}$ Department of Infectious Disease, The Fourth Affiliated Hospital of Anhui Medical University, Hefei, Anhui, People's Republic of China

\section{Video abstract}

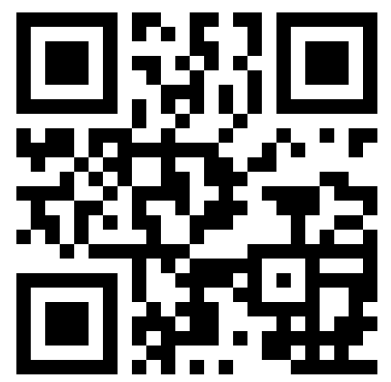

Point your SmartPhone at the code above. If you have a $Q R$ code reader the video abstract will appear. Or use: https://youtu.be/iftqWObPEIE
Correspondence: Ying Ye; Jiabin Li Tel +86-55I-62922713

Fax +86-55I-6292228I

Email yeying2@I39.com;

lijiabin@ahmu.edu.cn
Purpose: This study aimed to develop and validate a personalized prediction model of death risk in patients with Acinetobacter baumannii (A. baumannii) infection and thus guide clinical research and support clinical decision-making.

Patients and Methods: The development group is comprised of 350 patients with A. baumannii infection admitted between January 2013 and December 2015 in The First Affiliated Hospital of Anhui Medical University. Further, 272 patients in the validation group were admitted between January 2016 and December 2018. The univariate and multivariate logistic regression analyses were used to determine the independent risk factors for death with $A$. baumannii infection. The nomogram prediction model was established based on the regression coefficients. The discrimination of the proposed prediction model was evaluated using the area under the curve (AUC) of the receiver operating characteristic (ROC) curves and decision curve analysis (DCA). The calibration diagram was used to evaluate the calibration degree of this model.

Results: The infectious source, carbapenem-resistant A. baumannii (CRAB), hypoalbuminemia, Charlson comorbidity index (CCI), and mechanical ventilation (MV) were independent risk factors for death. The AUC of the ROC curve of the two groups was 0.768 and 0.792 , respectively. The net income was higher when the probability was between $30 \%$ and $80 \%$, showing a strong discrimination capacity of the proposed model. The calibration curve swung around the $45^{\circ}$ oblique line, indicating a high degree of calibration.

Conclusion: The proposed model helped predict the risk of death from A. baumannii infection, improve the early identification of patients with a higher risk of death, and guide clinical treatment.

Keywords: Acinetobacter baumannii, carbapenem resistance, prediction model, risk factors

\section{Introduction}

Acinetobacter baumannii (A. baumannii) is a gram-negative opportunistic bacillus with strict aerobic and nonlactose fermentation, which can cause a widespread nosocomial infection, especially in intensive care units (ICUs) and immunocompromised patients, including newborns. The most common manifestations are ventilator-associated pneumonia (VAP) and bacteremia, which can also cause meningitis, peritonitis, endocarditis, and urinary and skin infections. ${ }^{1-4} A$. baumannii infection accounts for about $2 \%$ of all health care-related infections in the United States and Europe, while the risk is even twice higher in Asia and the Middle East. ${ }^{5,6}$ The estimation statistics show that about $1,000,000$ cases of $A$. baumannii infection occur worldwide every year. It causes high 
mortality and morbidity, partially due to its resistance to many drugs, especially carbapenem resistance. ${ }^{7,8}$

With the improved understanding of $A$. baumannii, an increasing number of recent studies have focused on learning the independent predictors for A. baumannii infection. Some studies indicated that the severity of the disease, malignant tumors (especially hematological malignancies), and neutropenia have been shown to be risk factors for death from A. baumannii bacteremia. ${ }^{9,10}$ Shargian-Alon et al found that carbapenem-resistant $A$. baumannii (CRAB) bacteremia was associated with a very high risk of death. ${ }^{11}$ Ciginskiene and his colleagues found that sex, Sequential Organ Failure Assessment score of admission to ICU, and red blood cell transfusion were the risk factors for mortality from A. baumannii infection with VAP. ${ }^{12}$ However, most of the current studies focused on the analysis of risk factors for A. baumannii infection or death from $A$. baumannii infection in a single-system infection. ${ }^{11,13-16}$ Various infections caused by $A$. baumannii and death were observed in a large number of critically ill patients. Therefore, early detection of patients with high-risk factors for death and effective interventions can significantly reduce complications, thus improving the prognosis of patients.

Practical and accurate prediction tools can be used to predict the risk of death after $A$. baumannii infection. This study was novel in proposing an approach for predicting the risk of death from $A$. baumannii infection in various systems. It aimed to develop and validate a reliable and accurate risk prediction model, providing clues for screening a high risk of death caused by A. baumannii infection and guide appropriate clinical treatment.

\section{Patients and Methods}

\section{Patient Selection}

This study was carried out in The First Affiliated Hospital of Anhui Medical University (Quick-PJ 2020-03-10), a 2825-bed tertiary medical center in southeastern China. A total of 350 patients with $A$. baumannii infection were nonselectively and consecutively recruited between January 2013 and December 2015 in the development cohort, including 254 surviving and 96 dead patients. Moreover, 272 patients with A. baumannii infection were recruited between January 2016 and December 2018 in the validation group, including 200 surviving and 72 dead patients. The clinical data of the aforementioned patients were analyzed retrospectively, and the study was performed following the Guidelines for
Transparent Reporting of a multivariable model for Individual Prognosis or Diagnosis (The TRIPOD statement). ${ }^{17}$

\section{Inclusion Criteria}

The inclusion criteria were as follows:

1. Inpatients from January 2013 to December 2018 in The First Affiliated Hospital of Anhui Medical University.

2. Patients older than 18 years.

1. A.baumannii infections defined based on the criteria of the Centers for Disease Control and Prevention. ${ }^{18}$ A. baumannii infection was defined as the presence of clinical evidence of infection such as fever, chills, and leukocytosis, combined with the laboratory and radiological results at the same time. Clinical infections were acquired from different sources plus positive cultures of infected sites, such as respiratory tract, pleural fluid and ascites, cerebrospinal fluid (CSF), urine, blood, skin, and soft tissue. Patients received antibacterial treatment within at least $24 \mathrm{~h}$ of culture. Only the first $A$. baumannii infection for each patient was included in the study.

2. Sterile samples such as blood, CSF, and pleural fluid and ascites can be considered infected once the bacteria are cultured. However, nonsterile samples must meet the quality criteria, and quantitative culture should be used. Sputum specimen was considered to be qualified if it had $<10$ epithelial cells and $\geqq 25$ leukocytes/microscopic visual fields. The thresholds of lower respiratory tract infection in tracheal aspirate and bronchoalveolar lavage were $\geqq 10^{6} \mathrm{CFU} / \mathrm{mL}$ and $\geqq 10^{4} \mathrm{CFU} / \mathrm{mL}$, respectively. It was significant if the threshold for the mid-stream urine culture was $\geqq 10^{5} \mathrm{CFU} / \mathrm{mL}$ or $\geqq 10^{3} \mathrm{CFU} / \mathrm{mL}$ in the urinary catheter.

\section{Exclusion Criteria}

The exclusion criteria were as follows:

1. Repeated strains and contaminated samples isolated from the same patient

2. Noninfected or colonized patients, including patients who had no clinical symptoms, had no abnormal laboratory and/or radiological results, and did not receive antimicrobial therapy 
3. Mixed infection, meaning the simultaneous presence of other pathogens in addition to A. baumannii

4. Patients with missing data

\section{Data Collection}

Data on the following 10 factors of the participants were collected and analyzed: sex, age, infectious source (respiratory tract, pleural fluid and ascites, CSF, blood, urine, skin, and soft tissue), ICU admission history, CRAB, hospitalization history, anemia, hypoalbuminemia, Charlson comorbidity index $(\mathrm{CCI}){ }^{19}$ and mechanical ventilation (MV). Hospitalization history was defined as a history of hospitalization within 3 months before infection. Hemoglobin and albumin values were used to evaluate anemia and hypoalbuminemia on the day of positive microbiological culture (or within $24 \mathrm{~h}$ ). Anemia was defined as hemoglobin levels lower than $120 \mathrm{~g} / \mathrm{L}$ and $110 \mathrm{~g} / \mathrm{L}$ in men and women, respectively. Anemia was divided into mild, moderate, and severe degrees according to the hemoglobin value. The serum albumin level lower than 30 $\mathrm{g} / \mathrm{L}$ was defined as hypoalbuminemia. The severity of the disease was assessed using the CCI on the day of positive microbiological culture (or within $24 \mathrm{~h}$ ). Mortality was analyzed 30 days after $A$. baumannii isolation.

\section{Statistical Analysis}

In view of the short follow-up time, binary logistic regression was chosen. The aforementioned 10 variables were taken as independent variables, and the probability of patients dying within 30 days was considered as a dependent variable. The factors predictive of death from $A$. baumannii infection were identified using univariate regression analysis. The variables with $\mathrm{P}<0.05$ were incorporated in the subsequent multivariate logistic regression analysis, and the variables with $\mathrm{P}<0.05$ in multivariate analysis were finally included in the prediction model.

The nomogram prediction model was established based on the regression coefficients of independent variables. The development group consisted of patients infected with A. baumannii from 2013 to 2015 . This group was used to develop a nomogram predictive of death. Then, from 2016 to 2018, patients infected with A. baumannii were identified for a validation group, which was evaluated prospectively. We use full model and stepwise (stepAIC) selected model to build the model respectively.

The model performance was evaluated using the predictive accuracy for individual outcomes (discriminating ability) and the accuracy of point estimates of the function (calibration). The dichotomy result was typically evaluated by calculating the area under the curve (AUC) of the receiver operating characteristic (ROC) curve. ${ }^{20}$ In general, a prediction model with an AUC $>0.75$ indicated that the model showed an excellent degree of discrimination. ${ }^{21}$ However, AUC alone was not enough to show the model's ability in improving decision-making. ${ }^{22}$

With regard to clinical practicality, the net income was tested by the decision curve analysis (DCA). DCA was used to evaluate whether the nomogram was sufficiently robust for clinical practice. ${ }^{23}$ The net benefit was derived by calculating the difference between the true-positive rate and weighted false-positive rate across different threshold probabilities in the validation cohort. The "decision curve" was plotted against the threshold probability.

Calibration was assessed using a calibration plot, a graphic representation of the relationship between the observed outcome frequencies and the predicted probabilities. In a well-calibrated model, the predictions should fall on a 45-degree diagonal line.

All analyses were carried out using EmpowerStats (http://www.empowerstats.com) and the statistical software package $\mathrm{R}$, and the ROC curve, DCA curve, and calibration diagram were drawn. A $P$ value $<0.05$ showed that the difference was statistically significant.

\section{Ethics Statement}

The study was approved by the ethical research committee of The First Affiliated Hospital of Anhui Medical University (Quick-PJ 2020-03-10). The ethics committee approved the waiver of informed consent. The research data accessed were de-identified and anonymously analyzed. The study was conducted according to the Declaration of Helsinki.

\section{Results}

\section{Patient Demographics}

A total of 622 patients were included in this study. The development group comprised 350 patients, and the validation cohort comprised 272 patients (Table 1).

The comparison of baseline data showed no difference in sex, age, infectious source, ICU admission history, CRAB, hospitalization history, anemia, hypoalbuminemia, CCI, and MV between the development and validation groups.

\section{Nomogram Development}

The univariate regression analysis showed that the statistically significant risk factors included infectious source, ICU admission history, CRAB, hypoalbuminemia, CCI, and MV 
Table I Demographic and Clinical Characteristics of the Development and Validation Groups

\begin{tabular}{|c|c|c|c|}
\hline Group & $\begin{array}{l}\text { Development } \\
(n=350) \text { No. } \\
\text { of Patients (\%) }\end{array}$ & $\begin{array}{l}\text { Validation } \\
(n=272) \text { No. of } \\
\text { Patients (\%) }\end{array}$ & $P$ value \\
\hline $\begin{array}{l}\text { Sex } \\
\qquad \text { Male } \\
\text { Female }\end{array}$ & $\begin{array}{l}231(66.0) \\
119(34.0)\end{array}$ & $\begin{array}{l}195(71.7) \\
77(28.3)\end{array}$ & 0.130 \\
\hline $\begin{array}{l}\text { Age, year } \\
\quad>60 \\
\leqq 40 \\
41-60\end{array}$ & $\begin{array}{l}203(58.0) \\
39(11.1) \\
108(30.9)\end{array}$ & $\begin{array}{l}150(55.1) \\
22(8.1) \\
100(36.8)\end{array}$ & 0.195 \\
\hline $\begin{array}{l}\text { Infectious source } \\
\text { Respiratory tract } \\
\text { Pleural fluid and } \\
\text { ascites } \\
\text { CSF } \\
\text { Urine } \\
\text { Blood } \\
\text { Skin and soft tissue }\end{array}$ & $\begin{array}{l}278(79.4) \\
10(2.9) \\
7(2.0) \\
16(4.6) \\
13(3.7) \\
26(7.4)\end{array}$ & $\begin{array}{l}219(80.5) \\
12(4.4) \\
3(1.1) \\
9(3.3) \\
18(6.6) \\
11(4.0)\end{array}$ & 0.128 \\
\hline $\begin{array}{l}\text { ICU admission } \\
\text { history } \\
\text { Yes } \\
\text { No }\end{array}$ & $\begin{array}{l}200(57.1) \\
150(42.9)\end{array}$ & $\begin{array}{l}|6|(59.2) \\
\text { III (40.8) }\end{array}$ & 0.608 \\
\hline $\begin{array}{c}\text { CRAB } \\
\text { Yes } \\
\text { No }\end{array}$ & $\begin{array}{l}260(74.3) \\
90(25.7)\end{array}$ & $\begin{array}{l}201(73.9) \\
71(26.1)\end{array}$ & 0.913 \\
\hline $\begin{array}{l}\text { Hospitalization } \\
\text { history } \\
\text { No } \\
\text { Yes }\end{array}$ & $\begin{array}{l}200(57.1) \\
150(42.9)\end{array}$ & $\begin{array}{l}139(51.1) \\
133(48.9)\end{array}$ & 0.133 \\
\hline $\begin{array}{l}\text { Anemia } \\
\text { No } \\
\text { Mild } \\
\text { Moderate } \\
\text { Severe }\end{array}$ & $\begin{array}{l}\mid 20(34.3) \\
14 \mid(40.3) \\
84(24.0) \\
5(1.4)\end{array}$ & $\begin{array}{l}83(30.5) \\
122(44.9) \\
59(21.7) \\
8(2.9)\end{array}$ & 0.327 \\
\hline $\begin{array}{l}\text { Hypoalbuminemia } \\
\text { No } \\
\text { Yes }\end{array}$ & $\begin{array}{l}218(62.3) \\
132(37.7)\end{array}$ & $\begin{array}{l}185(68.0) \\
87(32.0)\end{array}$ & 0.138 \\
\hline $\begin{array}{l}\mathrm{CCl} \\
<4 \\
\geq 4\end{array}$ & $\begin{array}{l}296(84.6) \\
54(15.4)\end{array}$ & $\begin{array}{l}221(81.2) \\
51(18.8)\end{array}$ & 0.273 \\
\hline $\begin{array}{l}\text { MV } \\
\text { Yes } \\
\text { No }\end{array}$ & $\begin{array}{l}176(50.3) \\
174(49.7)\end{array}$ & $\begin{array}{l}156(57.3) \\
116(42.7)\end{array}$ & 0.080 \\
\hline
\end{tabular}

Abbreviations: $\mathrm{CCl}$, Charlson comorbidity index; $\mathrm{CRAB}$, carbapenem-resistant Acinetobacter baumannii; CSF, cerebrospinal fluid; ICUs, intensive care units; MV, mechanical ventilation.

$(P<0.05)$, while sex, age, hospitalization history, and anemia were not statistically significant in the development cohort. The statistically significant variables in the univariate regression analysis were included in multivariate logistic regression analysis. It was found that infectious source, CRAB, hypoalbuminemia, CCI, and MV were independent risk factors associated with death caused by $A$. baumannii infection (Table 2) $(P<0.05)$.

In the development group, the five variables with statistical significance in multivariate analysis, which were also included in the prediction model, played different roles in the impact on mortality. The fatality rate varied with different sources of infection. Among them, the mortality associated with blood and CSF infections was the highest $(69.2 \%$ and $57.1 \%$, respectively). The second causes of mortality were respiratory tract infection and pleural fluid and ascites ( $28.1 \%$ and $30 \%$, respectively). The mortality due to skin and soft tissue infections was low (7.7\%), and none of the patients with urinary tract infections died. The mortality of patients with CRAB (33.8\%) was much higher than the mortality of patients infected by carbapenem-sensitive Acinetobacter baumannii (CSAB) (8.9\%). In addition, the mortality of patients with hypoalbuminemia and of patients with CCI $\geqq 4$ was as high as $39.4 \%$ and $44.4 \%$, respectively, while the mortality of those without hypoproteinemia and CCI $<4$ was $20.2 \%$ and $24.3 \%$, respectively. The mortality of patients with MV (40.3\%) was much higher than that of patients without MV (14.4\%). The mortality rates of the other five variables are shown in Table 2.

A personalized nomogram was established to predict the risk of death from A. baumannii infection (Figure 1). For plotting the nomogram, first, the point index corresponding to each prediction was obtained, the total score was recorded, and then the total risk score was predicted by computing the probability related to the risk of death from A. baumannii infection.

We present the specific regression model that underlies this nomogram. It was found that the full model and stepwise (stepAIC) selected model presented the same model.

$\operatorname{logit}(\mathrm{Y})=-0.93800+0.70982 *($ Infectious source $=$ Pleural fluid and ascites) $+1.71349 *$ (Infectious source $=$ CSF) $-15.09755 *($ Infectious source $=$ Urine $)+1.50138 *$ (Infectious source $=$ Blood $)-1.12139 *($ Infectious source $=$ Skin and soft tissue) $-0.93628 *(\mathrm{CRAB}=\mathrm{No})+0.73636 *$ (Hypoalbuminemia $=$ Yes) $\quad+0.89659 *(\mathrm{CCI} \geq 4) \quad-0.93462^{*}$ (MV=No)

Figure 1 presents the nomogram for predicting the death rate of patients with A. baumannii infection. By pointing the straight line vertically up to the "point" axis, each clinical factor corresponded to a specific point. After the sum point was on the axis of the total point, the sum 
Table 2 Univariate and Multivariate Logistic Regression Models in the Development Group

\begin{tabular}{|c|c|c|c|c|c|c|c|}
\hline & \multicolumn{3}{|c|}{ Univariate Analysis } & \multicolumn{3}{|c|}{ Multivariate Analysis } & \multirow[t]{2}{*}{ Mortality (\%) } \\
\hline & OR & $95 \% \mathrm{Cl}$ & $P$ & OR & $95 \% \mathrm{Cl}$ & $P$ & \\
\hline \multicolumn{8}{|l|}{ Sex } \\
\hline Male & 1.0 & & & & & & 65/23I (28.I) \\
\hline Female & 0.90 & $0.55-1.48$ & 0.678 & & & & $31 / 119(26.1)$ \\
\hline \multicolumn{8}{|l|}{ Age (year) } \\
\hline$>60$ & 1.0 & & & & & & $58 / 203(28.6)$ \\
\hline$\leqq 40$ & 1.56 & $0.77-3.19$ & 0.220 & & & & $15 / 39(38.5)$ \\
\hline $4 I-60$ & 0.68 & $0.39-1.18$ & 0.165 & & & & $23 / 108(2 \mid .3)$ \\
\hline \multicolumn{8}{|l|}{ Infectious source } \\
\hline Respiratory tract & 1.0 & & & 1.0 & & & $78 / 278(28.1)$ \\
\hline Pleural fluid and ascites & 1.10 & $0.28-4.36$ & 0.893 & 2.13 & $0.45-10.04$ & $0.34 I$ & $3 / 10(30.0)$ \\
\hline CSF & 3.42 & $0.75-15.63$ & 0.113 & 5.49 & $1.07-28.14$ & 0.041 & $4 / 7(57.1)$ \\
\hline Urine & 0.00 & $0.00-\operatorname{lnf}$ & 0.979 & 0.00 & $0.00-\operatorname{lnf}$ & 0.988 & $0 / 16(0)$ \\
\hline Blood & 5.77 & $1.73-19.28$ & 0.004 & 4.64 & $1.26-17.06$ & 0.021 & $9 / 13(69.2)$ \\
\hline Skin and soft tissue & 0.21 & $0.05-0.93$ & 0.039 & 0.31 & $0.07-1.44$ & 0.136 & $2 / 26(7.7)$ \\
\hline \multicolumn{8}{|l|}{ ICU admission history } \\
\hline Yes & 1.0 & & & 1.0 & & & $73 / 200(36.5)$ \\
\hline No & 0.32 & $0.19-0.53$ & $<0.001$ & 1.41 & $0.56-3.55$ & 0.465 & $23 / 150(15.3)$ \\
\hline \multicolumn{8}{|l|}{ CRAB } \\
\hline Yes & 1.0 & & & 1.0 & & & $88 / 260(33.8)$ \\
\hline No & 0.19 & $0.09-0.41$ & $<0.001$ & 0.36 & $0.15-0.90$ & 0.029 & $8 / 90(8.9)$ \\
\hline \multicolumn{8}{|l|}{ Hospitalization history } \\
\hline No & 1.0 & & & & & & $54 / 200(27)$ \\
\hline Yes & 1.05 & $0.65-1.69$ & 0.836 & & & & $42 / 150(28)$ \\
\hline \multicolumn{8}{|l|}{ Anemia } \\
\hline No & 1.0 & & & & & & $28 / 120(23.3)$ \\
\hline Mild & 1.08 & $0.6 \mathrm{I}-1.92$ & 0.779 & & & & $35 / I 4 I(24.8)$ \\
\hline Moderate & 1.83 & $0.99-3.38$ & 0.055 & & & & $30 / 84(35.7)$ \\
\hline Severe & 4.93 & $0.78-30.99$ & 0.089 & & & & $3 / 5(60)$ \\
\hline \multicolumn{8}{|l|}{ Hypoalbuminemia } \\
\hline No & 1.0 & & & 1.0 & & & $44 / 218(20.2)$ \\
\hline Yes & 2.57 & $1.59-4.16$ & $<0.001$ & 2.04 & I.19-3.48 & 0.009 & $52 / 132(39.4)$ \\
\hline \multicolumn{8}{|l|}{$\mathrm{CCl}$} \\
\hline$<4$ & 1.0 & & & 1.0 & & & $72 / 296(24.3)$ \\
\hline$\gg 4$ & 2.49 & $1.37-4.53$ & 0.003 & 2.57 & $1.31-5.04$ & 0.006 & $24 / 54(44.4)$ \\
\hline \multicolumn{8}{|l|}{ MV } \\
\hline Yes & 1.0 & & & 1.0 & & & $71 / 176(40.3)$ \\
\hline No & 0.25 & $0.15-0.42$ & $<0.001$ & 0.31 & $0.13-0.75$ & 0.009 & $25 / 174(14.4)$ \\
\hline
\end{tabular}

Abbreviations: $\mathrm{CCl}$, Charlson comorbidity index; $\mathrm{Cl}$, confidence internal; CRAB, carbapenem-resistant Acinetobacter baumannii; CSF, cerebrospinal fluid; ICUs, intensive care units; MV, mechanical ventilation; OR, odds ratio.

was drawn directly to the death axis to indicate the probability of death after infection. For example, the cumulative score of various predictive indicators for a patient with CRAB infection (10.7 points), positive blood culture
(97.5 points), serum albumin count lower than $30 \mathrm{~g} / \mathrm{L}$ (8.5 points), CCI $\geq 4$ (10.3 points), and treatment without MV ( 0 points) was $10.7+97.5+8.5+10.3+0=127$. Thus, the corresponding predicted risk of death for the patient 

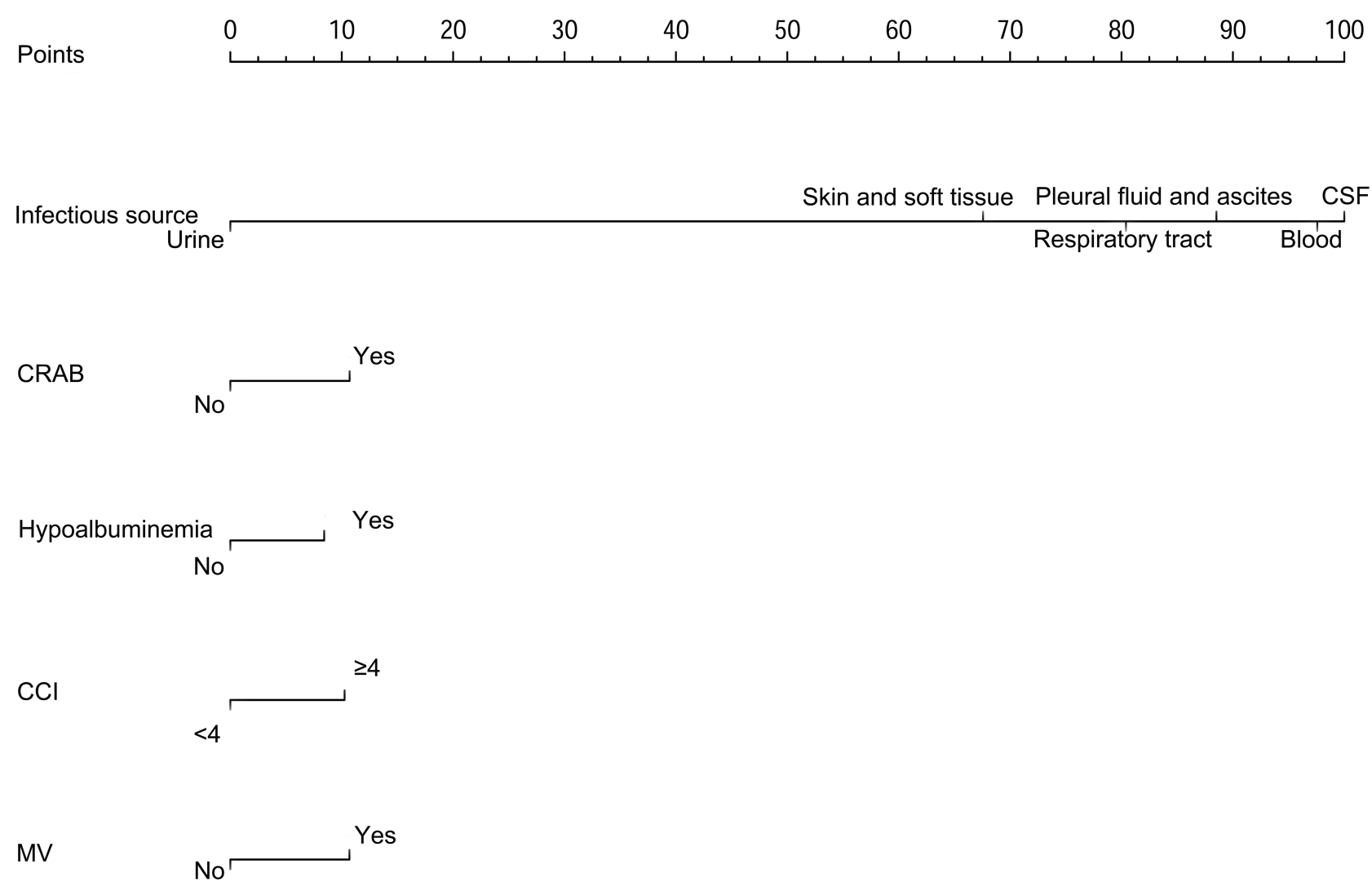

Total points

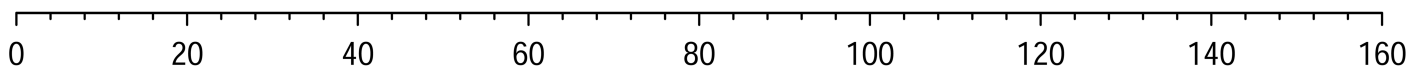

Linear predictor

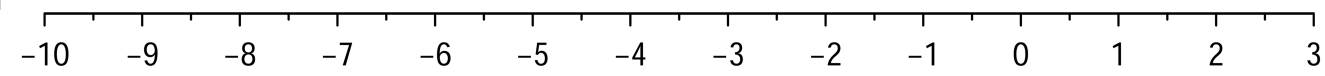

Risk of death

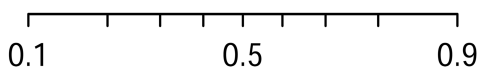

Figure I Nomogram to predict the probability of risk of death from A. baumannii infection.

Abbreviations: $\mathrm{CCl}$, Charlson comorbidity index; CRAB, carbapenem-resistant Acinetobacter baumannii; CSF, cerebrospinal fluid; MV, mechanical ventilation.

was 0.78 (78\%) (Figure 2). The predicted risk value was then used in the decision-making of treatment planning and patient consultation (Figure 2).

\section{Nomogram Validation}

The discrimination and calibration degree were used to validate the prediction model. The AUC value for the risk of death in the development and validation groups was 0.768 and 0.792 (Figure 3), respectively. The ROC curve analysis of development group and validation group is shown in Table 3 . The net income of the development and validation groups was higher when the probability was between $30 \%$ and $80 \%$ (Figure 4 ). This showed that the prediction model had a perfect discrimination ability. The calibration curve swung around the $45^{\circ}$ oblique line, indicating a high degree of calibration (Figure 5).

\section{Discussion}

The mortality of study patients in the development and validation groups was 27.4\% (96/350) and 26.5\% (72/272), respectively, consistent with the results of two Spanish 


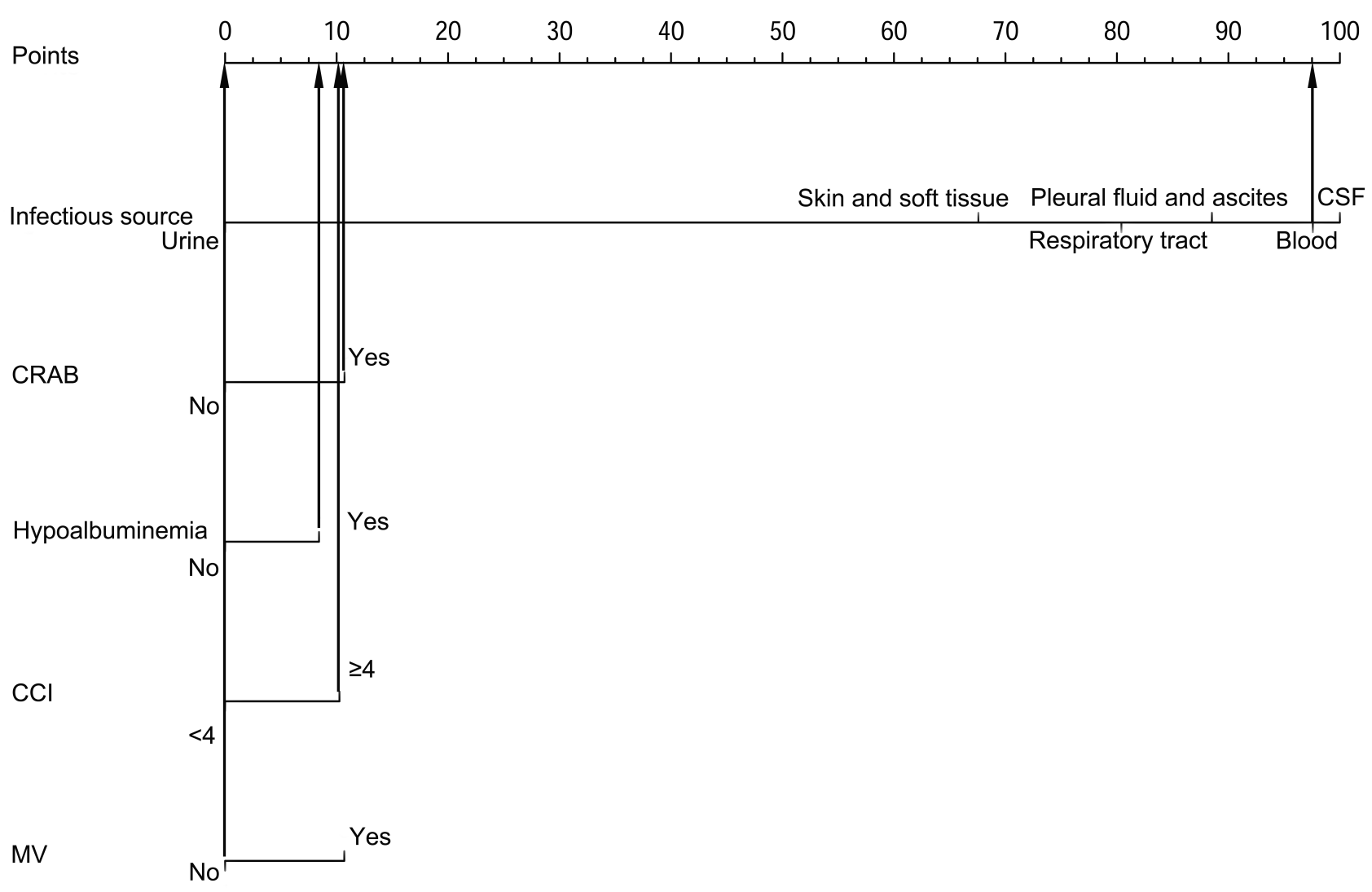

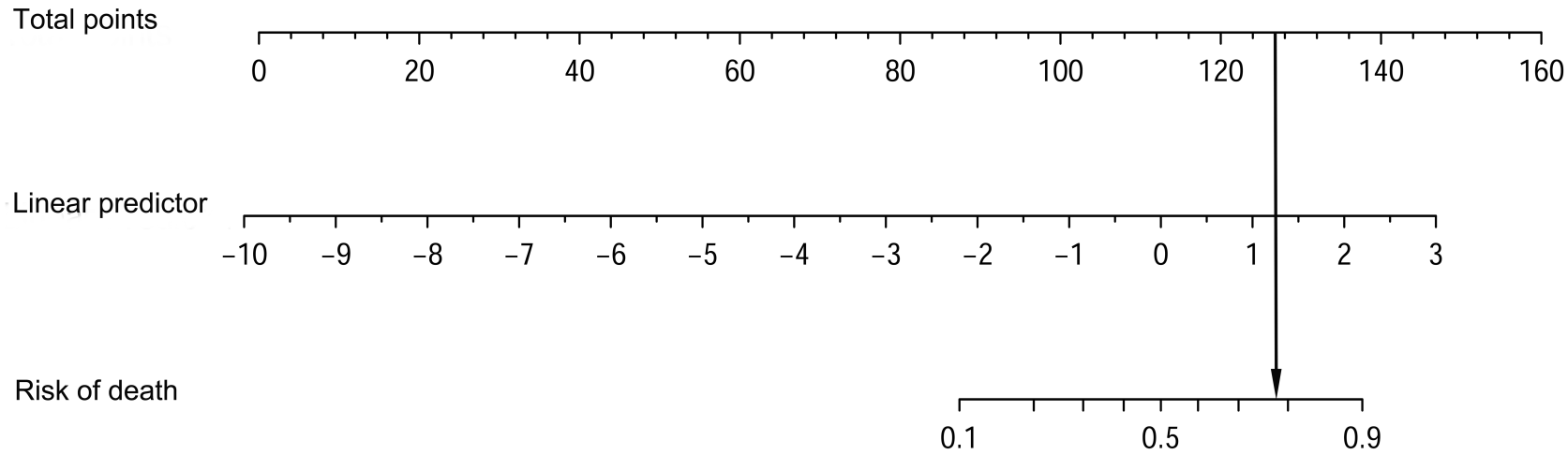

Figure 2 Example of prediction nomogram for the risk of death from A. baumannii infection.

Abbreviations: $\mathrm{CCl}$, Charlson comorbidity index; CRAB, carbapenem-resistant Acinetobacter baumannii; CSF, cerebrospinal fluid; MV, mechanical ventilation.

multicenter studies and a multicenter study in the United Kingdom. ${ }^{24-26}$ A recent meta-analysis also found that the overall mortality rate of A. baumannii infection was $33 \%{ }^{27}$

This study found that patients with $A$. baumannii infection from the blood and CSF had significantly higher scores in the predictive model compared with patients infected from other sources. In recent years, bloodstream infections caused by $A$. baumannii have been increasingly observed in patients hospitalized in ICUs and general wards. ${ }^{28-32}$ Bacteremia is an important and common cause of death in patients. ${ }^{33}$ The total mortality of patients with $A$. baumannii bacteremia ranged from $29 \%$ to $83.7 \%{ }^{29-32}$ Brotfain et al showed that patients with VAP and secondary A. baumannii bacteremia had higher ICU mortality compared with those with nonbacteremic $A$. baumannii $\mathrm{VAP}^{28}$

The harmfulness of intracranial infection caused by A. baumannii has attracted increasing attention of researchers in recent years. The literature review by Kim et al showed that the all-cause mortality of Acinetobacter meningitis was $15-71 \%{ }^{34}$ A recent study on children with meningitis 


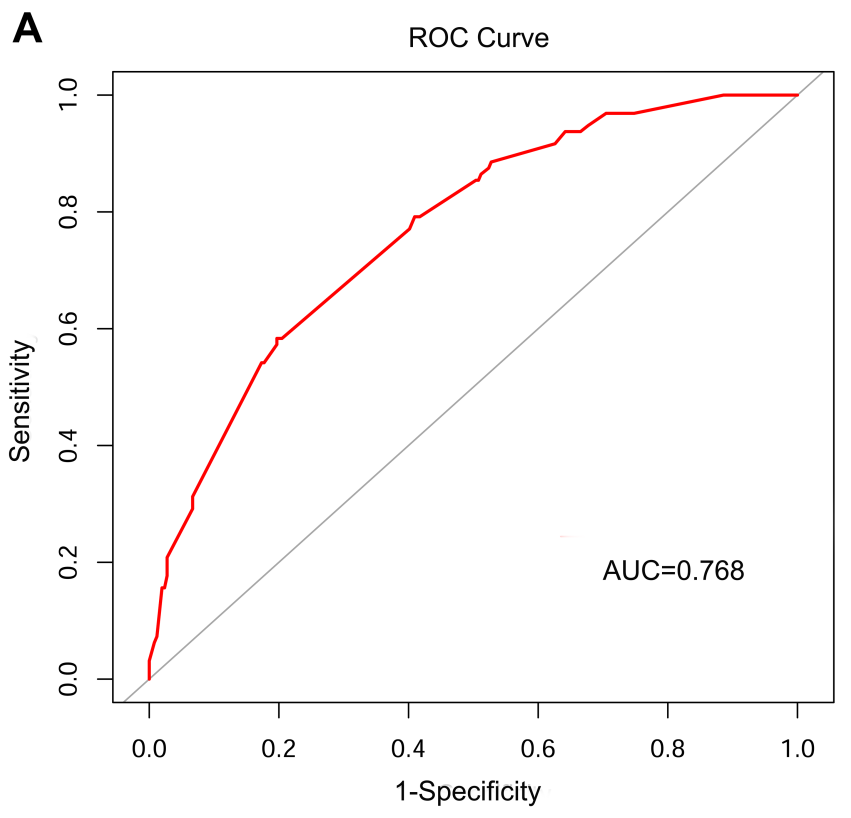

B

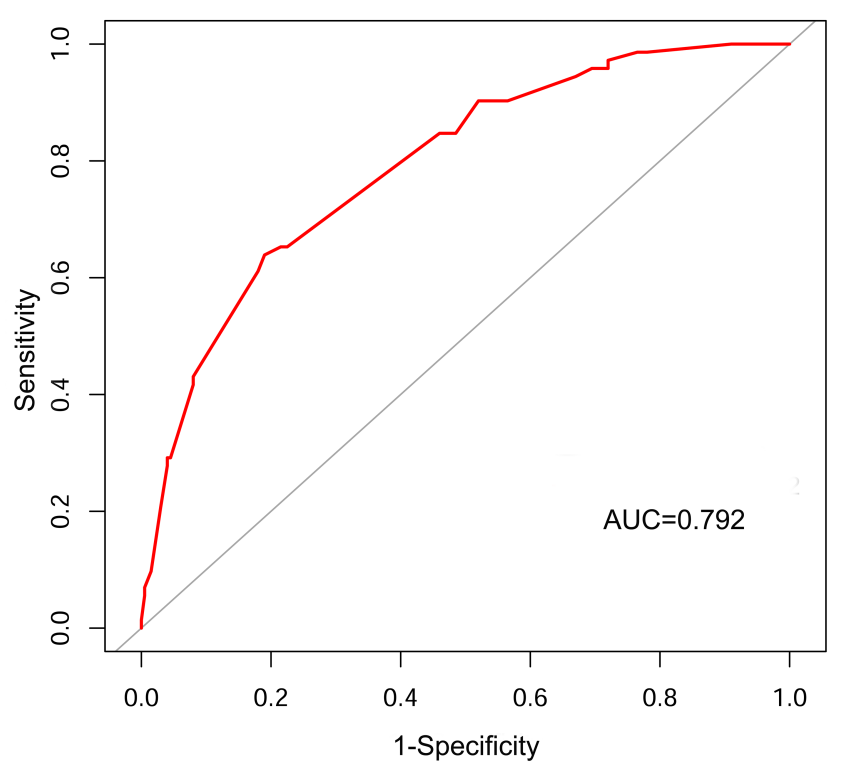

Figure 3 ROC curves for validating the discrimination of the nomogram. (A) Development group (B) Validation group (AUC $=0.768$ vs 0.792 ). Abbreviations: AUC, area under the curve; ROC, receiver operating characteristic.

infected by A. baumannii found that the case fatality rate of children was close to $50 \%{ }^{35}$ The high mortality rate of intracranial infection was mainly due to the difficulty in the infiltration of antibiotics through the blood-brain barrier and drug resistance. ${ }^{36}$ One basic study showed that most of the strains isolated from sputum showed high biofilm-forming ability. However, strains isolated from body fluids, such as

Table 3 ROC Curve Analysis of the Development Group and Validation

\begin{tabular}{|l|l|l|}
\hline Group & $\begin{array}{l}\text { Development }(\mathbf{n}= \\
\mathbf{3 5 0})\end{array}$ & $\begin{array}{l}\text { Validation } \mathbf{( n}= \\
\mathbf{2 7 2})\end{array}$ \\
\hline Death & 96 & 72 \\
Survive & 254 & 200 \\
ROC & 0.768 & 0.792 \\
$95 \% \mathrm{Cl}$. low & 0.715 & 0.733 \\
$95 \% \mathrm{Cl}$. upp & 0.821 & 0.851 \\
Specificity & 0.803 & 0.810 \\
Sensitivity & 0.583 & 0.639 \\
Accuracy & 0.743 & 0.765 \\
Positive predictive & 0.528 & 0.548 \\
value & & \\
Negative predictive & 0.836 & 0.862 \\
value & & \\
a & 56 & 46 \\
b & 50 & 38 \\
c & 40 & 26 \\
d & 204 & 162 \\
\hline
\end{tabular}

Abbreviations: $\mathrm{Cl}$, confidence interval; $\mathrm{ROC}$, receiver operating characteristic. blood and CSF, showed high serum resistance. ${ }^{37}$ This might, to some extent, explain the contribution of specimens from different sources to the death in the prediction model.

In this study, CRAB was another factor in predicting the risk of death from A. baumannii infection. Recognizing the growing importance of this species, the World Health Organization has listed CRAB as a key priority pathogen in the priority list of drug-resistant bacteria. ${ }^{38}$ The data of China Antimicrobial Surveillance Network showed that the resistance rate of $A$. baumannii to imipenem and meropenem increased year by year from $31 \%$ and $39 \%$ in 2005 to $77.1 \%$ and $78.1 \%$ in 2018 , respectively. ${ }^{39}$ A meta-analysis showed that the mortality of patients with CRAB infection was higher than that of patients with CSAB infection. ${ }^{27}$ This might be due to the higher severity of the disease and inappropriate empirical antibiotic treatment in patients with CRAB. In the study by Wang and his colleagues, only about one third of the isolates were CRAB. Hence, the 30day mortality rate was low, indicating that carbapenem resistance was one of the important factors for the risk of death from $A$. baumannii infection. ${ }^{40}$

$\mathrm{CCI} \geqq 4$ was also found as an important risk factor associated with death caused by $A$. baumannii infection. Alessandro Russo and colleagues found that the $\mathrm{CCI}>3$ was a risk factor for 14-day mortality from $A$. baumannii bloodstream infection. ${ }^{14}$ Martin-Aspas et al found that CCI was significantly lower in the survival group than in the death group. $^{16}$ 

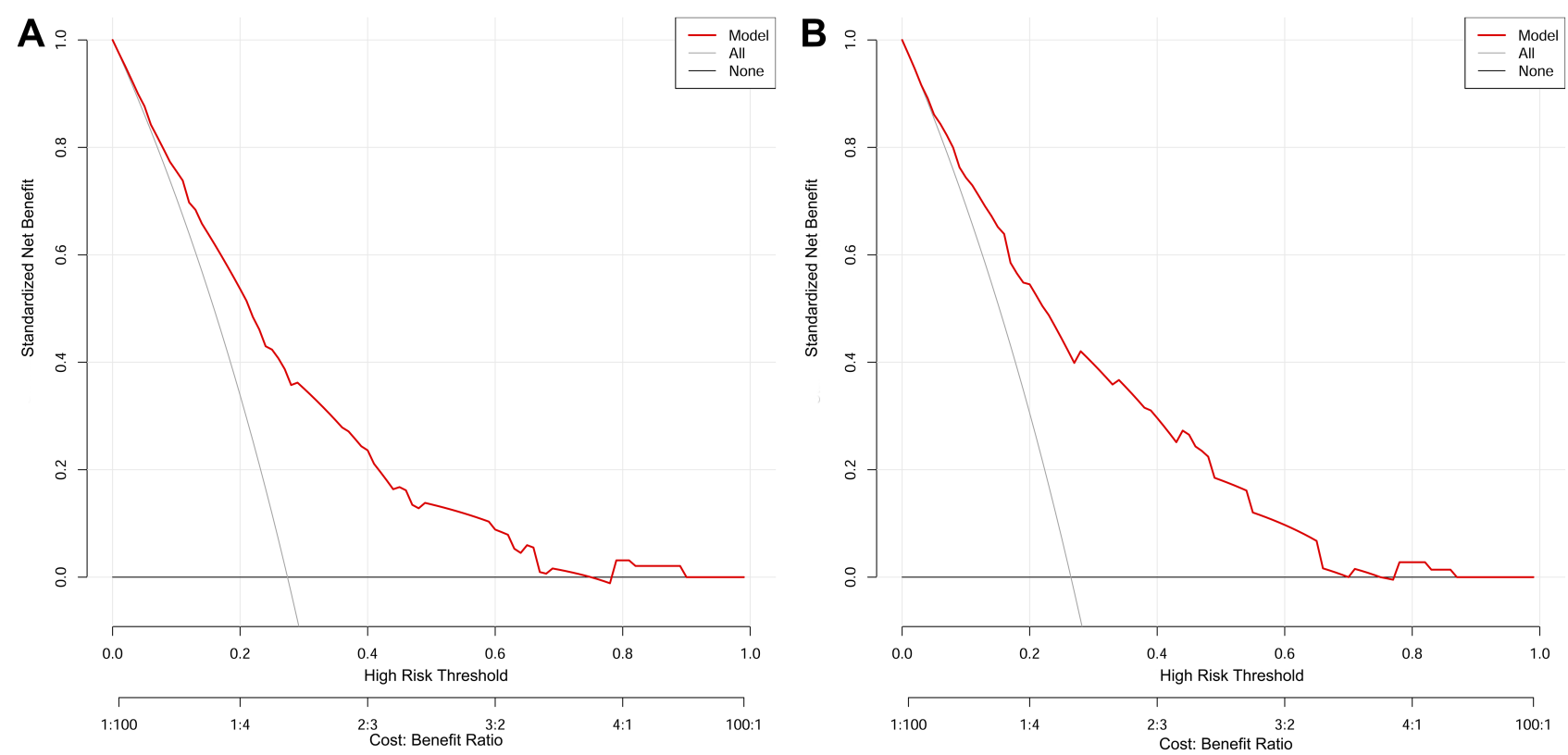

Figure 4 DCA curves for validating the net income of the nomogram. (A) Development group (B) Validation group. Abbreviation: DCA, decision curve analysis.
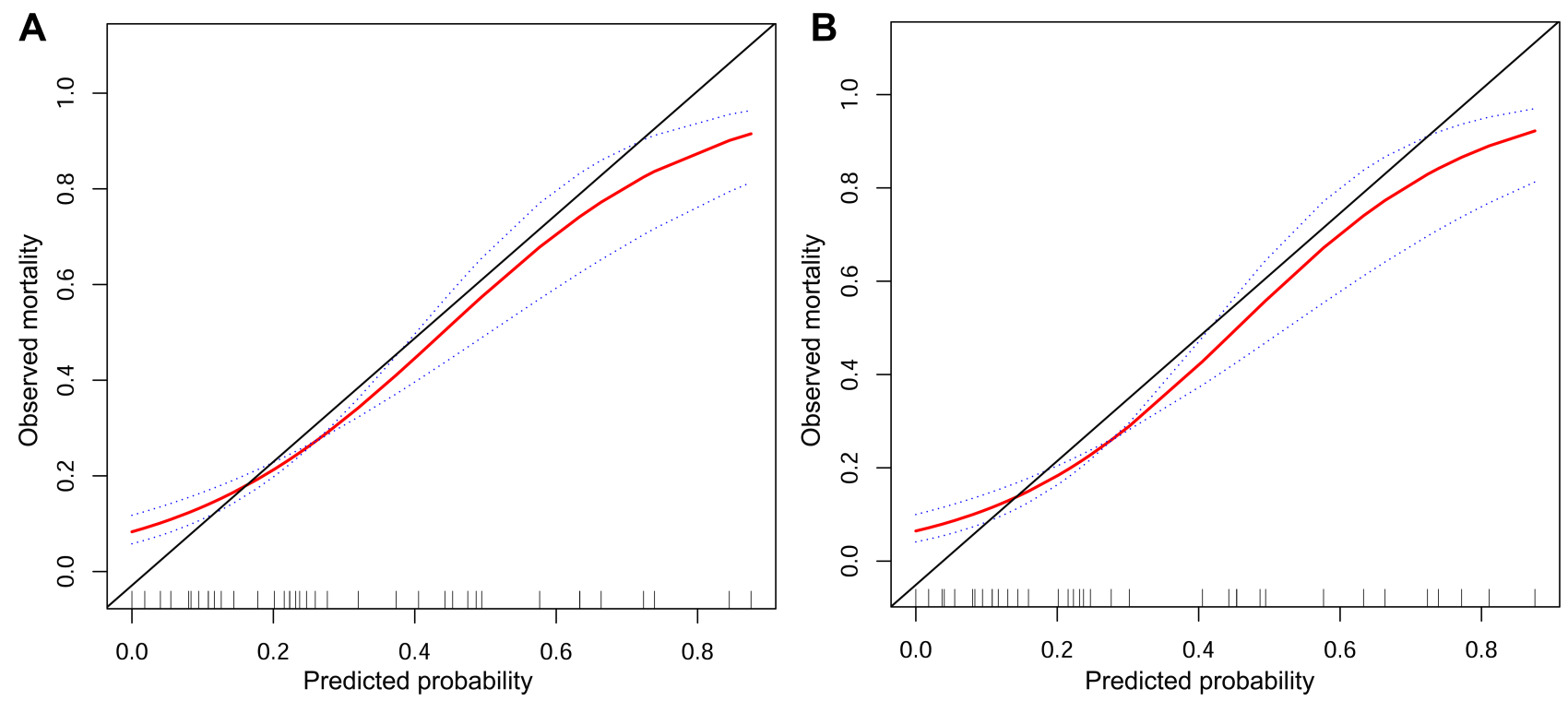

Figure 5 Calibration curves for validating the calibration of the nomogram. (A) Development group (B) Validation group.

Previous studies found an important relationship between MV and A. baumannii infection. ${ }^{41,42}$ The present study also found that MV was another independent risk factor for the death from $A$. baumannii infection. ${ }^{43-45}$ However, in the univariate analysis, MV was found to be a protective factor for the risk of death from $A$. baumannii infection at baseline, which might be explained by the bias of better prognosis of ventilated patients at baseline. ${ }^{11}$

This study also suggested that hypoalbuminemia was closely related to death caused by A. baumannii infection.
Some studies found that hypoalbuminemia before transplantation was an important risk factor for multidrugresistant $A$. baumannii infection in patients with lung transplantation. ${ }^{15}$ Hypoalbuminemia was also found to be an independent risk factor for respiratory tract infection of A. baumannii in ICU. ${ }^{46}$ Hypoalbuminemia is generally considered as an indicator of weakness and homeostasis instability in the body. ${ }^{47}$ Hypoalbuminemia is believed to be a risk factor for death in patients infected with A. baumannii, which might be related to low immunity 
and critical condition, susceptibility to infection, and death after infection.

As a presentation form of a prediction model, nomogram is a useful complementary tool to help clinicians make clinical decisions. The nomogram developed in this study can help clinicians judge the prognosis of patients with $A$. baumannii infection as soon as possible based on existing clinical data and make clinical treatment in time.

This study had several strengths. First, this study was novel in predicting the risk of death from A. baumannii infection. Second, the characteristics of clinical infection of A. baumannii were comprehensive, and many studies did not distinguish infection or colonization. This study excluded asymptomatic carriers and patients with mixed bacterial infections to exclude colonized patients as much as possible. Third, the study sample size was large, and the scope of patient study not only included ICU patients but also expanded to the general ward outside ICU.

This study had certain limitations as well. First, this was a retrospective study, and since the patient's information was obtained from the electronic medical record system, unrecorded variables could not be analyzed, and selection bias could not be avoided. A comprehensive clinical evaluation of the infection could not be made, and the physician's clinical judgment was only considered. However, the inclusion and exclusion criteria were strictly formulated in this study, and sufficient clinical samples were collected to minimize the potential bias. Of course, further prospective studies should be carried out to validate the findings in the future. Second, this was a single-center study, which might have impacted the generalizability of the results. Patient data in different periods from the same facility were used to validate the model; it was persuasive to compare some randomly selected samples in a center. However, this still belonged to internal validation, while external verification was more convincing. The model was validated to use additional data sets from other facilities, regions, and countries. In the future, a multicenter predictive model study in conjunction with other medical institutions should be conducted to improve the feasibility of the predictive model. Third, it was impossible to distinguish between infection and colonization in mixed infection, and thus the case data of mixed infection were excluded, leading to selection bias. Finally, the purpose of the study was to provide a risk prediction model for death associated with A. baumannii for all types of infections. However, in the present study, respiratory infections were strongly overrepresented and accounted for $79.4 \%$ of the total samples. In general, in other related studies, the number of samples of respiratory tract infection was indeed higher than that of other sources of infection. ${ }^{10,16,25}$ We nonselectively and consecutively recruited case data, and data are true and reliable. This selection bias might be due to the exclusion of data of mixed infection, or it might be the bias brought about by a single-center study. Therefore, our model is not suitable for other facility with different infection rates.Future studies should include more data from other institutions to increase the number of samples from other sources of infection.

\section{Conclusions}

In summary, an individualized prediction model was developed and validated in this study for predicting the risk of death from A. baumannii infection in our facility, thus helping improve the early identification of patients with a higher risk of death and guiding appropriate clinical treatment. Prospective multicenter studies are needed to improve the universality of this model.

\section{Abbreviations}

A. baumannii, Acinetobacter baumannii; AUC, Area under the curve; CCI, Charlson comorbidity index; CRAB, Carbapenem-resistant Acinetobacter baumannii; CSAB, Carbapenem-sensitive Acinetobacter baumannii; CSF, Cerebrospinal fluid; DCA, Decision curve analysis; ICUs, Intensive care units; MV, Mechanical ventilation; ROC, Receiver operating characteristic; VAP, Ventilatorassociated pneumonia.

\section{Acknowledgments}

The authors acknowledge the contributions of doctors and staff of the medical record room along with the microbiology laboratory and the patients in The First Affiliated Hospital of Anhui Medical University to this study.

\section{Funding}

This study was supported by the National Natural Science Foundation of China (Grant No. 81373072).

\section{Disclosure}

The authors report no conflicts of interest related to this study.

\section{References}

1. Antunes LC, Visca P, Towner KJ. Acinetobacter baumannii: evolution of a global pathogen. Pathog Dis. 2014;71:292-301. doi:10. 1111/2049-632X.12125 
2. Fournier PE, Richet H. The epidemiology and control of Acinetobacter baumannii in health care facilities. Clin Infect Dis. 2006;42:692-699. doi:10.1086/500202

3. Harding CM, Hennon SW, Feldman MF. Uncovering the mechanisms of Acinetobacter baumannii virulence. Nat Rev Microbiol. 2018;16:91-102. doi:10.1038/nrmicro.2017.148

4. Ulu-Kilic A, Gundogdu A, Cevahir F, Kilic H, Gunes T, Alp E. An outbreak of bloodstream infection due to extensively resistant Acinetobacter baumannii among neonates. Am J Infect Control. 2018;46:154-158. doi:10.1016/j.ajic.2017.08.007

5. Magill SS, Edwards JR, Bamberg W, et al. Multistate point-prevalence survey of health care-associated infections. $N$ Engl $J$ Med. 2014;370:1198-1208. doi:10.1056/NEJMoa1306801

6. Lob SH, Hoban DJ, Sahm DF, Badal RE. Regional differences and trends in antimicrobial susceptibility of Acinetobacter baumannii. Int $J$ Antimicrob Agents. 2016;47:317-323. doi:10.1016/j.ijantimicag.2016.01.015

7. Spellberg B, Rex JH. The value of single-pathogen antibacterial agents. Nat Rev Drug Discov. 2013;12:963. doi:10.1038/nrd3957c1

8. Birgani MT, Ansari H. Antibiotic Characterization of Acinetobacter baumannii isolated from clinical samples and production of recombinant OmpA from resistant strains. Jundishapur J Microbiol. 2018;11:e78773.

9. Gu Z, Han Y, Meng T, et al. Risk factors and clinical outcomes for patients with Acinetobacter baumannii bacteremia. Medicine (Baltimore). 2016;95:e2943. doi:10.1097/MD.0000000000002943

10. Nazer LH, Kharabsheh A, Rimawi D, Mubarak S, Hawari F. Characteristics and outcomes of Acinetobacter baumannii infections in critically ill patients with cancer: a matched case-control study. Microb Drug Resist. 2015;21:556-561. doi:10.1089/ mdr.2015.0032

11. Shargian-Alon L, Gafter-Gvili A, Ben-Zvi H, et al. Risk factors for mortality due to Acinetobacter baumannii bacteremia in patients with hematological malignancies - a retrospective study. Leuk Lymphoma. 2019;60:2787-2792. doi:10.1080/10428194.2019.1599113

12. Ciginskiene A, Dambrauskiene A, Rello J, Adukauskiene D. Ventilator-associated pneumonia due to drug-resistant Acinetobacter baumannii: risk factors and mortality relation with resistance profiles, and independent predictors of in-hospital mortality. Medicina (Kaunas). 2019;55:49.

13. Jang TN, Lee SH, Huang CH, Lee CL, Chen WY. Risk factors and impact of nosocomial Acinetobacter baumannii bloodstream infections in the adult intensive care unit: a case-control study. $J$ Hosp Infect. 2009;73:143-150. doi:10.1016/j.jhin.2009.06.007

14. Russo A, Bassetti M, Ceccarelli G, et al. Bloodstream infections caused by carbapenem-resistant Acinetobacter baumannii: clinical features, therapy and outcome from a multicenter study. $J$ Infect. 2019;79:130-138. doi:10.1016/j.jinf.2019.05.017

15. Oh DH, Kim YC, Kim EJ, et al. Multidrug-resistant Acinetobacter baumannii infection in lung transplant recipients: risk factors and prognosis. Infect Dis (Lond). 2019;51:493-501. doi:10.1080/ 23744235.2018.1556400

16. Martin-Aspas A, Guerrero-Sanchez FM, Garcia-Colchero F, Rodriguez-Roca S, Giron-Gonzalez JA. Differential characteristics of Acinetobacter baumannii colonization and infection: risk factors, clinical picture, and mortality. Infect Drug Resist. 2018;11:861-872. doi:10.2147/IDR.S163944

17. Collins GS, Reitsma JB, Altman DG, Moons KG. Transparent reporting of a multivariable prediction model for individual prognosis or diagnosis (TRIPOD): the TRIPOD statement. $B r \quad J$ Surg. 2015;102:148-158. doi:10.1002/bjs.9736

18. Horan TC, Andrus M, Dudeck MA. CDC/NHSN surveillance definition of health care-associated infection and criteria for specific types of infections in the acute care setting. Am J Infect Control. 2008;36:309-332. doi:10.1016/j.ajic.2008.03.002
19. Charlson ME, Pompei P, Ales KL, MacKenzie CR. A new method of classifying prognostic comorbidity in longitudinal studies: development and validation. J Chronic Dis. 1987;40:373-383. doi:10.1016/ 0021-9681(87)90171-8

20. Harrell FE Jr., Califf RM, Pryor DB, Lee KL, Rosati RA. Evaluating the yield of medical tests. JAMA. 1982;247:2543-2546. doi:10.1001/ jama.1982.03320430047030

21. Niu XK, He WF, Zhang Y, et al. Developing a new PI-RADS v2-based nomogram for forecasting high-grade prostate cancer. Clin Radiol. 2017;72:458-464. doi:10.1016/j.crad.2016.12.005

22. Holmberg L, Vickers A. Evaluation of prediction models for decision-making: beyond calibration and discrimination. PLoS Med. 2013;10:e1001491. doi:10.1371/journal.pmed.1001491

23. Vickers AJ, Elkin EB. Decision curve analysis: a novel method for evaluating prediction models. Med Decis Making. 2006;26:565-574. doi:10.1177/0272989X06295361

24. Villar M, Cano ME, Gato E, et al. Epidemiologic and clinical impact of Acinetobacter baumannii colonization and infection: a reappraisal. Medicine (Baltimore). 2014;93:202-210. doi:10.1097/MD.00000 00000000036

25. Lopez-Cortes LE, Cisneros JM, Fernandez-Cuenca F, et al. Monotherapy versus combination therapy for sepsis due to multidrug-resistant Acinetobacter baumannii: analysis of a multicentre prospective cohort. J Antimicrob Chemother. 2014;69:3119-3126. doi:10.1093/jac/dku233

26. Livermore DM, Hill RL, Thomson H, et al. Antimicrobial treatment and clinical outcome for infections with carbapenem- and multiply-resistant Acinetobacter baumannii around London. Int J Antimicrob Agents. 2010;35:19-24. doi:10.1016/j.jjantimicag.2009.09.014

27. Lemos EV, de la Hoz FP, Einarson TR, et al. Carbapenem resistance and mortality in patients with Acinetobacter baumannii infection: systematic review and meta-analysis. Clin Microbiol Infect. 2014;20:416-423. doi:10.1111/1469-0691.12363

28. Brotfain E, Borer A, Koyfman L, et al. Multidrug resistance Acinetobacter bacteremia secondary to ventilator-associated pneumonia: risk factors and outcome. $J$ Intensive Care Med. 2017;32:528-534. doi:10.1177/0885066616632193

29. Wisplinghoff H, Bischoff T, Tallent SM, Seifert H, Wenzel RP, Edmond MB. Nosocomial bloodstream infections in US hospitals: analysis of 24,179 cases from a prospective nationwide surveillance study. Clin Infect Dis. 2004;39:309-317. doi:10.1086/421946

30. Nutman A, Glick R, Temkin E, et al. A case-control study to identify predictors of 14-day mortality following carbapenem-resistant Acinetobacter baumannii bacteraemia. Clin Microbiol Infect. 2014;20:O1028-1034. doi:10.1111/1469-0691.12716

31. Ballouz T, Aridi J, Afif C, et al. Risk factors, clinical presentation, and outcome of Acinetobacter baumannii Bacteremia. Front Cell Infect Microbiol. 2017;7:156. doi:10.3389/fcimb.2017.00156

32. Freire MP, de Oliveira Garcia D, Garcia CP, et al. Bloodstream infection caused by extensively drug-resistant Acinetobacter baumannii in cancer patients: high mortality associated with delayed treatment rather than with the degree of neutropenia. Clin Microbiol Infect. 2016;22:352-358. doi:10.1016/j.cmi.2015.12.010

33. Levy MM, Artigas A, Phillips GS, et al. Outcomes of the surviving sepsis campaign in intensive care units in the USA and Europe: a prospective cohort study. Lancet Infect Dis. 2012;12:919-924. doi:10.1016/S1473-3099(12)70239-6

34. Kim BN, Peleg AY, Lodise TP, et al. Management of meningitis due to antibiotic-resistant Acinetobacter species. Lancet Infect Dis. 2009;9:245-255. doi:10.1016/S1473-3099(09)70055-6

35. Xiao J, Zhang C, Ye S. Acinetobacter baumannii meningitis in children: a case series and literature review. Infection. 2019;47:643-649. doi:10.1007/s15010-018-1234-1

36. Cascio A, Conti A, Sinardi L, et al. Post-neurosurgical multidrug-resistant Acinetobacter baumannii meningitis successfully treated with intrathecal colistin. A new case and a systematic review of the literature. Int $J$ Infect Dis. 2010;14:e572-579. doi:10.1016/j.jij.2009.06.032 
37. Shirazi AS, Shafiei M, Solgi H, Aslani MM, Azizi O, Badmasti F. Different virulence capabilities and ompA expressions in ST2 and ST513 of multidrug-resistant Acinetobacter baumannii.. Curr Microbiol. 2019;76:723-731. doi:10.1007/s00284-019-01686-9

38. World Health Organization. Global priority list of antibiotic-resistant bacteria to guide research, discovery, and development of new antibiotics. Available from: http://www.who.int/medicines/publica tions/WHO-PPL-Short_Summary_25Feb-ET_NM_WHO.pdf. Accessed November 11, 2018.

39. Hu F, Guo Y, Yang Y, et al. Resistance reported from China antimicrobial surveillance network (CHINET) in 2018. Eur J Clin Microbiol Infect Dis. 2019;38(12):2275-2281.

40. Wang X, Zhang L, Sun A, et al. Acinetobacter baumannii bacteraemia in patients with haematological malignancy: a multicentre retrospective study from the Infection Working Party of Jiangsu Society of Hematology. Eur J Clin Microbiol Infect Dis. 2017;36:1073-1081. doi:10.1007/s10096-016-2895-2

41. Abbo A, Navon-Venezia S, Hammer-Muntz O, Krichali T, SiegmanIgra Y, Carmeli Y. Multidrug-resistant Acinetobacter baumannii. Emerg Infect Dis. 2005;11:22-29. doi:10.3201/eid1101.040001

42. Arvaniti K, Lathyris D, Ruimy R, et al. The importance of colonization pressure in multiresistant Acinetobacter baumannii acquisition in a Greek intensive care unit. Crit Care. 2012;16:R102. doi:10.1186/ cc11383
43. Erbay A, Idil A, Gozel MG, Mumcuoglu I, Balaban N. Impact of early appropriate antimicrobial therapy on survival in Acinetobacter baumannii bloodstream infections. Int $J$ Antimicrob Agents. 2009;34:575-579. doi:10.1016/j.ijantimicag.2009.07.006

44. de Gouvea EF, Martins IS, Halpern M, et al. The influence of carbapenem resistance on mortality in solid organ transplant recipients with Acinetobacter baumannii infection. BMC Infect Dis. 2012;12:351. doi:10.1186/1471-2334-12-351

45. Yang S, Sun J, Wu X, Zhang L. Determinants of mortality in patients with Nosocomial Acinetobacter baumannii Bacteremia in Southwest China: a five-year case-control study. Can J Infect Dis Med Microbiol. 2018;2018:3150965. doi:10.1155/2018/3150965

46. Ushizawa H, Yahata $\mathrm{Y}$, Endo $\mathrm{T}$, et al. A Epidemiological Investigation of a Nosocomial Outbreak of Multidrug-Resistant Acinetobacter baumannii in a Critical Care Center in Japan, 2011-2012. Jpn J Infect Dis. 2016;69:356. doi:10.7883/yoken. JJID.2016.E002

47. Van Hemelrijck M, Harari D, Garmo H, et al. Biomarker-based score to predict mortality in persons aged 50 years and older: a new approach in the Swedish AMORIS study. Int $J$ Mol Epidemiol Genet. 2012;3:66-76.

\section{Publish your work in this journal}

Infection and Drug Resistance is an international, peer-reviewed openaccess journal that focuses on the optimal treatment of infection (bacterial, fungal and viral) and the development and institution of preventive strategies to minimize the development and spread of resistance. The journal is specifically concerned with the epidemiology of antibiotic resistance and the mechanisms of resistance development and diffusion in both hospitals and the community. The manuscript management system is completely online and includes a very quick and fair peerreview system, which is all easy to use. Visit http://www.dovepress.com/ testimonials.php to read real quotes from published authors. 